\title{
Magnesium isoglycyrrhizinate has hepatoprotective effects in an oxaliplatin-induced model of liver injury
}

\author{
XUEQING ZOU ${ }^{1,2}$, YONGMEI WANG ${ }^{3}$, CHENG PENG $^{1}$, BEN WANG $^{1}$, \\ ZHENGCHUAN NIU ${ }^{1,4}$, ZEQUN LI ${ }^{1,4}$ and JUN NIU ${ }^{1}$ \\ ${ }^{1}$ Department of General Surgery, Qilu Hospital, Shandong University, Jinan, Shandong 250012; \\ ${ }^{2}$ Department of Anesthesiology; ${ }^{3}$ Center of Diagnosis and Treatment of Breast Disease, The Affiliated Hospital \\ of Qingdao University, Qingdao, Shandong 266000; ${ }^{4}$ Key Laboratory of Cardiovascular Remodeling and \\ Function Research, Chinese Ministry of Education and Public Health, Jinan, Shandong 250012, P.R. China
}

Received February 7, 2018; Accepted July 5, 2018

DOI: $10.3892 / \mathrm{ijmm} .2018 .3787$

\begin{abstract}
Oxaliplatin is a core chemotherapeutic agent used for the treatment of colorectal liver metastasis; however, liver injury caused by oxaliplatin increases the risk of peri-operative morbidity and mortality. Magnesium isoglycyrrhizinate (MgiG) is a magnesium salt of 18- $\alpha$ glycyrrhizic acid stereoisomer that has demonstrated liver-protective effects against toxins and hepatitis. In the present study, the liver-protective effect of MgiG against oxaliplatin-induced hepatic injury was examined in vitro and in vivo. The results demonstrated that MgiG had a protective effect against oxaliplatin-induced liver injury, as evidenced by the alleviation of hepatic pathological damage and transaminase levels. The protective effect of MgiG was demonstrated to be correlated with inhibition of oxidative stress, the interleukin- 6 pathway and the coagulation system. Altogether, the present findings suggested that MgiG may have potential value in the clinical prevention and treatment of oxaliplatin-induced liver injury.
\end{abstract}

\section{Introduction}

Colon cancer is the third most common cancer and the fourth leading cause of cancer-related death worldwide (1). The presence of liver metastasis at early stages, even at the time of diagnosis, is the predominant reason. The use of adjuvant chemotherapy for colorectal liver metastases (CRLM) has increased resection rates and improved outcomes $(2,3)$. Oxaliplatin is one of the most commonly used chemotherapeutic agents. Studies have revealed that after six months of

Correspondence to: Dr Jun Niu, Department of General Surgery, Qilu Hospital, Shandong University, 107 Wenhuaxi Street, Jinan, Shandong 250012, P.R. China

E-mail: niujunsdu@163.com

Key words: oxaliplatin-associated liver injury, magnesium isoglycyrrhizinate, oxidative stress, interleukin-6, coagulation system oxaliplatin-based chemotherapy, patients with potentially resectable liver metastases showed significantly improved overall and disease-free survival rates (4-6).

However, oxaliplatin-induced liver injury is a limiting factor for the otherwise highly effective use of the drug in patients with CRLM (7). In on-going studies, it has been proved that there is oxaliplatin-associated hepatotoxicity in a considerable portion of patients $(8,9)$. Liver injury leads to an increased risk of peri-operative morbidity and mortality in liver resection cases (10). Previous global gene microarray analyses have demonstrated that oxaliplatin-induced liver injury correlated with elevated oxidative stress, activation of the interleukin-6 (IL-6) pathway and overactivation of the coagulation cascade $(11,12)$. To overcome these side-effects, a medication that protects the liver against damage caused by oxaliplatin is urgently needed.

Magnesium isoglycyrrhizinate (MgiG) is a magnesium salt of 18- $\alpha$ glycyrrhizic acid stereoisomer. The full name of MgiG is tetrahydrate magnesium $18 \alpha, 20 \beta$-hydroxy-11-oxon orolean-12-en-3 $\beta$-yl-2-O- $\beta$-D-glucopyranurosyl- $\alpha$-D-glucopy ranosiduronate (13). Previous studies have demonstrated that MgiG attenuates liver injury caused by toxins (carbon tetrachloride and D-galactosamine) and other factors, including free fatty acid, ethanol and ischemia/reperfusion (14). MgiG exerts a greater liver-protecting effect than glycyrrhizin or $\beta$-glycyrrhizic acid (15-17). Recently, there have been reports of MgiG possessing hepatoprotective effects against chemotherapeutic agents, such as cyclophosphamide, by attenuating oxidative stress and cytokines, such as IL-6, in liver cells (18). A recent in vivo study reported that reduced glutathione alleviates oxaliplatin-induced acute liver injury (19). However, there has not been a study regarding whether MgiG could be used for the prevention or treatment of oxaliplatin-associated liver injury.

In the present study, the protective functions of MgiG against oxaliplatin-induced liver injury were investigated in vitro and in vivo. To better imitate the pathogenic process of oxaliplatin-induced liver injury, a liver damage animal model was established by direct oxaliplatin administration, where the drug was administered to animals based on a treatment schedule comparable to human patients receiving 
chemotherapy. The mechanisms underlying the hepatoprotective effects of MgiG were also investigated, including oxidative stress, inflammatory responses and the coagulation cascade.

\section{Materials and methods}

Reagents. Magnesium isoglycyrrhizinate (MgiG) was purchased from Chia Tai Tianqing Pharmaceutical Group Co., Ltd. (Jiangsu, China). Oxaliplatin was obtained from Sigma-Aldrich (Merck KGaA, Darmstadt, Germany). ELISA kits for IL-6 (KGEMC138) and von Willebrand factor (vWF) (KGEMC146) were obtained from Nanjing KeyGEN Biotech. Co., Ltd. (Nanjing, China). The alanine aminotransferase (ALT), aspartate aminotransferase (AST), glutathione (GSH) and malondialdehyde (MDA) detection kits were obtained from Nanjing Jiancheng Bioengineering Institute (Nanjing, China). Unless indicated otherwise, chemicals used in experiments were purchased from Sigma-Aldrich (Merck KGaA).

Animals and treatments. Forty male, 10-week-old C57Bl/6 mice, weighing 18-22 g, were obtained from the Laboratory Animal Center of the School of Medicine, Shandong University (Shandong, China). Animals were kept under standard conditions of $55 \%$ humidity, $20-25^{\circ} \mathrm{C}$, and a controlled 12-h light/dark cycle, and allowed free access to standard food and water. All C57Bl/6 mice were randomly divided into 4 groups. Group I mice were intraperitoneally (i.p.) injected with vehicle (normal saline) as control $(\mathrm{n}=10)$. Group II mice received $10 \mathrm{mg} / \mathrm{kg}$ intraperitoneal oxaliplatin on a weekly basis for 5 weeks $(\mathrm{n}=10)$. Group III mice received an low dose of MgiG [15 mg/kg/day, intraperitoneal (i.p.)] together with intraperitoneal oxaliplatin $(n=10)$. Group IV mice received high-dose $\mathrm{MgiG}(45 \mathrm{mg} / \mathrm{kg} /$ day, i.p.) together with intraperitoneal oxaliplatin $(n=10)$. The drug dosing schedule was based on previously published studies and our preliminary experiments $(18,20-22)$. Mice were sacrificed one week following the final dose of chemotherapy under isoflurane anesthesia by cardiac puncture. The present study was conducted in accordance with Chinese laws and National Institute of Health publications on the use and care of laboratory animals and using a protocol approved by the Institutional Animal Care and Use Committee of Shandong University (Shandong, China).

Cell culture. The human hepatic cell line LO2 and the human colon cancer cell line HT-29 were purchased from the American Type Culture Collection (Manassas, VA, USA). The cells were maintained in RPMI1640 medium for LO2 (Gibco; Thermo Fisher Scientific, Inc., Waltham, MA, USA) and DMEM for HT-29 (Gibco; Thermo Fisher Scientific, Inc.) containing 10\% fetal bovine serum (Gibco; Thermo Fisher Scientific, Inc.), $100 \mathrm{U} / \mathrm{ml}$ penicillin, and $100 \mu \mathrm{g} / \mathrm{ml}$ streptomycin (Amresco, Inc.; VWR International, Radnor, PA, USA) at $37^{\circ} \mathrm{C}$ in a $5 \%$ $\mathrm{CO}_{2}$ humidified atmosphere.

Analysis of liver enzymes and other indicators. AST, ALT, GSH and MDA commercial kits were used to measure the indicators, according to the manufacturers' instructions. In brief, liver homogenates, serum or cell supernatant substrate solutions were added to the reacting system, and the final products were spectrophotometrically measured with various wavelengths. The concentrations of the indicator were calculated according to standard curves. The concentrations of IL-6 and vWF in serum and liver homogenates were measured with ELISA kits, according to the manufacturer's instructions.

Cell Counting Kit-8 (CCK-8) assay for cell viability. Cell viability was determined with a CCK- 8 assay. Briefly, cells were plated in 96-well plates at a density of 3,000 cells per well. After $10 \mathrm{~h}$, the medium was replaced with fresh medium containing $300 \mu \mathrm{M}$ oxaliplatin, with or without other reagents. The doses of oxaliplatin and other reagents were determined based on previous studies and our preliminary experiments $(18,23)$. Experimental groups were treated with various concentrations of MgiG. Untreated cells were used as control. Following incubation for $24 \mathrm{~h}$, the cells were exposed to CCK-8 (100 $\mu \mathrm{l} / \mathrm{ml}$; Dojindo Molecular Technologies, Inc., Kumamoto, Japan) followed by incubation at $37^{\circ} \mathrm{C}$ for another $2 \mathrm{~h}$. Subsequently, the absorbance of the resulting color in each well was measured using a microplate spectrophotometer at a wavelength of $450 \mathrm{~nm}$.

Histological assessment. Formalin-fixed and paraffin-embedded liver tissues were cut into $5 \mu \mathrm{m}$-thick slices. Hematoxylin and eosin (H\&E) and Sirius red stains were used. The pathological changes of both stained slices were evaluated by a pathologist blinded to the experimental group. The histological changes were scored according to the following criteria: 0 , absent; 1 , mild; 2 , moderate; and 3 , severe.

$R N A$ extraction and reverse transcription-quantitative polymerase chain reaction (RT-qPCR) analysis. Total RNA of liver homogenates and cells was extracted and purified using TRIzol reagent (Thermo Fisher Scientific, Inc.). The reverse transcription of $1 \mu \mathrm{g}$ RNA to cDNA was established using the PrimeScript RT reagent kit (cat. no. RR036A; Takara Bio, Inc., Otsu, Japan). qPCR was run on a Bio-Rad iQ5 optical module (Bio-Rad Laboratories, Inc., Hercules, CA, USA). Cycling conditions were: $95^{\circ} \mathrm{C}$ for $2 \mathrm{~min}$ as initial denaturation, 40 cycles of denaturation at $95^{\circ} \mathrm{C}$ for $15 \mathrm{sec}$, and annealing/extension at $60^{\circ} \mathrm{C}$ for $40 \mathrm{sec}$. Melt curve analysis was set between 65 and $95^{\circ} \mathrm{C}$ with $0.5^{\circ} \mathrm{C}$ increments at $5 \mathrm{sec}$ per step. Quantitative values were obtained by the threshold cycle $(\mathrm{Cq})$ value. Relative mean fold change in expression ratios was calculated by the $2^{-\Delta \Delta \mathrm{Cq}}$ method (24). GAPDH was used as internal control. The primer sequences used in the present study were as follows: GAPDH, forward 5'-GCACAG TCAAGGCCGAGAAT-3' and reverse 5'-GCCTTCTCCATG GTGGTGAA-3'; metallothionein 1 (Mt1), forward 5'-GCT GCTGCTCCTGCTGTCCC-3' and reverse 5'-CAGCACGTG CACTTGTCCGC-3'; peroxiredoxin 1 (PRDX1), forward 5'-TGTTTCCCCAGCATGTGTACC-3' and reverse 5'-TGC TCTTATAGAAGACCCAGGTTC-3'; superoxide dismutase 2 (SOD2), forward 5'-CGTGACTTTGGGTCTTTTGAG-3' and reverse 5'-TAGAGCAGGCAGCAATCTGTAA-3'; IL-6, forward 5'-GAGGATACCACTCCCAACAGACC-3' and reverse 5'-AAGTGCATCATCGTTGTTCATACA-3'; signal transducer and activator of transcription 3 (STAT3), forward 5'-CCTTCTTGTTCTACGGCTTGC-3' and reverse 5'-TCG CCTATCTTCTCAACCAGG-3'; vWF, forward 5'-CGG 
GAAGAGTGTGATGGTTGAC-3' and reverse 5'-AGC ATCTCCCACAGCATTCACC-3'; plasminogen activator inhibitor-1 (PAI-1), forward 5'-GATGCTATGGGATTCAAA GTCA-3' and reverse 5'-TCCACCTGTTTCACCATAGTCT-3'.

Protein extraction and western blotting. Mice liver samples or cells were lysed in radioimmunoprecipitation assay lysis solution (Beyotime Institute of Biotechnology, Haimen, China) containing phosphatase inhibitors and PMSF. A BCA Protein Assay kit (Beyotime Institute of Biotechnology) was used to determine the protein concentrations. A total of $30 \mu \mathrm{g}$ protein extracts were resolved by 8 and 15\% SDS-PAGE. The proteins were transferred onto polyvinylidene difluoride membranes (EMD Millipore, Billerica, MA, USA), blocked with 5\% nonfat dry milk at room temperature for $1 \mathrm{~h}$ and incubated with primary antibodies at $4^{\circ} \mathrm{C}$ overnight. The following antibodies were used: Metallothionein 1 (cat. no. ab12228; 1:2,000; Abcam, Cambridge, MA, USA), peroxiredoxin 1 (cat. no. 8499; 1:5,000; Cell Signaling Technology, Inc., Danvers, MA, USA), superoxide dismutase 2 (cat. no. 13141; 1:500; Cell Signaling Technology, Inc.), STAT3 (cat. no. 9132; 1:1,000; Cell Signaling Technology, Inc.); vWF (cat. no. 65707; 1:500; Cell Signaling Technology, Inc.), PAI-1 (cat. no. 11907; 1:2,000; Cell Signaling Technology, Inc.). and GAPDH (cat. no. ab22555; 1:2,500; Abcam). The blots were then incubated with horseradish peroxidase-conjugated secondary antibodies at room temperature for $1.5 \mathrm{~h}$ (cat. nos. BA1054 and BA1050; 1:50,000; Wuhan Boster Biological Technology, Ltd., Wuhan, China), and visualized by enhanced chemiluminescence detection. GAPDH was used as the loading control. The optical density was analyzed with ImageJ 10.0 software (National Institutes of Health, Bethesda, MD, USA).

Statistical analysis. Three independent repeats were performed for each experiment. Experimental data were expressed as the mean \pm standard deviation. Analyses were performed using GraphPad Prism 5 (GraphPad Software, Inc., La Jolla, CA, USA). Comparisons among datasets were made using one-way analysis of variance followed by Tukey's post hoc test or unpaired Student's t-test. $\mathrm{P}<05$ was considered to indicate a statistically significant difference.

\section{Results}

MgiG attenuates the aminotransferase increase caused by oxaliplatin. Aminotransferase levels are diagnostic markers of liver damage. $\mathrm{LO} 2$ cells exposed to oxaliplatin were cultured in medium supplemented with or without MgiG, and mice receiving oxaliplatin were treated with or without MgiG. AST and ALT activity in serum and cell supernatants was then measured. Following oxaliplatin challenge, both indicators significantly increased in cell supernatants (Fig. 1A). In the MgiG-treated groups, the increase in indicators was significantly attenuated (Fig. 1A). AST and ALT levels continued decreasing when the MgiG concentration was below $100 \mu \mathrm{M}$. AST and ALT activity in mouse serum also displayed a significant increase following oxaliplatin administration (Fig. 1B). MgiG effectively attenuated the oxaliplatin-induced changes and this effect was dose-dependent with a more significant reduction observed in the high-dose group (Fig. 1B).
MgiG improves oxaliplatin-induced pathological changes in liver parenchyma. To assess the histological changes in liver parenchyma, H\&E-stained liver sections were blindly reviewed by a pathologist and graded according to the degree of histological damage. A large area of hepatocyte necrosis, extensive vacuolization, and inflammatory cell infiltration were observed in the oxaliplatin-treated group (Fig. 1C). MgiG treatment significantly attenuated the pathological damage in the liver of the experimental animals (Fig. 1C). High-dose MgiG had a more significant effect in protecting the liver against oxaliplatin-induced damage (Fig. 1C). Sirius red staining was also performed on the tissue sections to estimate fibrosis in the liver. Collagen deposition was observed within the injured hepatic sinusoids in the liver of oxaliplatin-treated mice (Fig. 1D). This pathological progress was significantly attenuated in the liver of MgiG-treated mice (Fig. 1D).

MgiG improves cell viability after oxaliplatin challenge in hepatic cells. A cell viability assay was performed on LO2 cells to estimate their response to oxaliplatin challenge and treatment of MgiG at various concentrations. Cell viability decreased by $57 \%$ in the oxaliplatin-treated group compared with the control group (Fig. 2A). There was an increase in cell viability in MgiG-treated groups (Fig. 2A). When the concentration of MgiG was $>100 \mu \mathrm{M}$, the rise in viability occurred in a dose-dependent manner (Fig. 2A). The same experiment was repeated using the colon cancer cell line HT-29. Cell viability also decreased dramatically following oxaliplatin exposure, but MgiG treatment had no effect in the oxaliplatin-mediated loss of viability in the colon cancer cells (Fig. 2B).

MgiG protects liver cells by attenuating oxaliplatin-induced oxidative stress. It has been well-demonstrated in previous studies that increased oxidative stress is one of the major mechanisms inducing sinusoidal obstruction syndrome (SOS) in patients and animals receiving oxaliplatin treatment $(11,21,25,26)$. To estimate oxidative stress, contents of MDA and GSH in liver homogenates and cell supernatants were measured (27). In LO2 cell supernatants, the content of MDA significantly increased following oxaliplatin exposure, and MgiG treatment significantly attenuated MDA levels (Fig. 2C). In liver homogenates from experimental mice, MDA increased 5.04-fold following oxaliplatin administration, and MgiG treatment remarkably attenuated this effect (Fig. 2D). In addition, a remarkable reduction in the concentration of GSH in LO2 cell supernatants was observed with oxaliplatin exposure, and MgiG significantly restored this (Fig. 2E). In vivo, the GSH levels in the liver homogenates of oxaliplatin-injected mice decreased by 59\% compared with control, and $\mathrm{MgiG}$ treatment significantly increased the GSH levels by 74 and $113 \%$ at low and high-dose, respectively (Fig. 2F).

To explore the role of oxidative stress in the pathogenic process of oxaliplatin-induced liver injury and the mechanism of the protective function of MgiG, a cell viability assay was performed on $\mathrm{LO} 2$ cells in the presence of supplementary $1 \mathrm{mM} \mathrm{H}_{2} \mathrm{O}_{2}$ or $100 \mu \mathrm{M}$ GSH in the medium. The dose of $\mathrm{H}_{2} \mathrm{O}_{2}$ for creating oxidative stress in vitro was based on previous studies and our preliminary experiments (28-30). When $\mathrm{H}_{2} \mathrm{O}_{2}$ was added in the medium, cell viability decreased by $34 \%$ in the oxaliplatin group, while high-dose MgiG treatment 

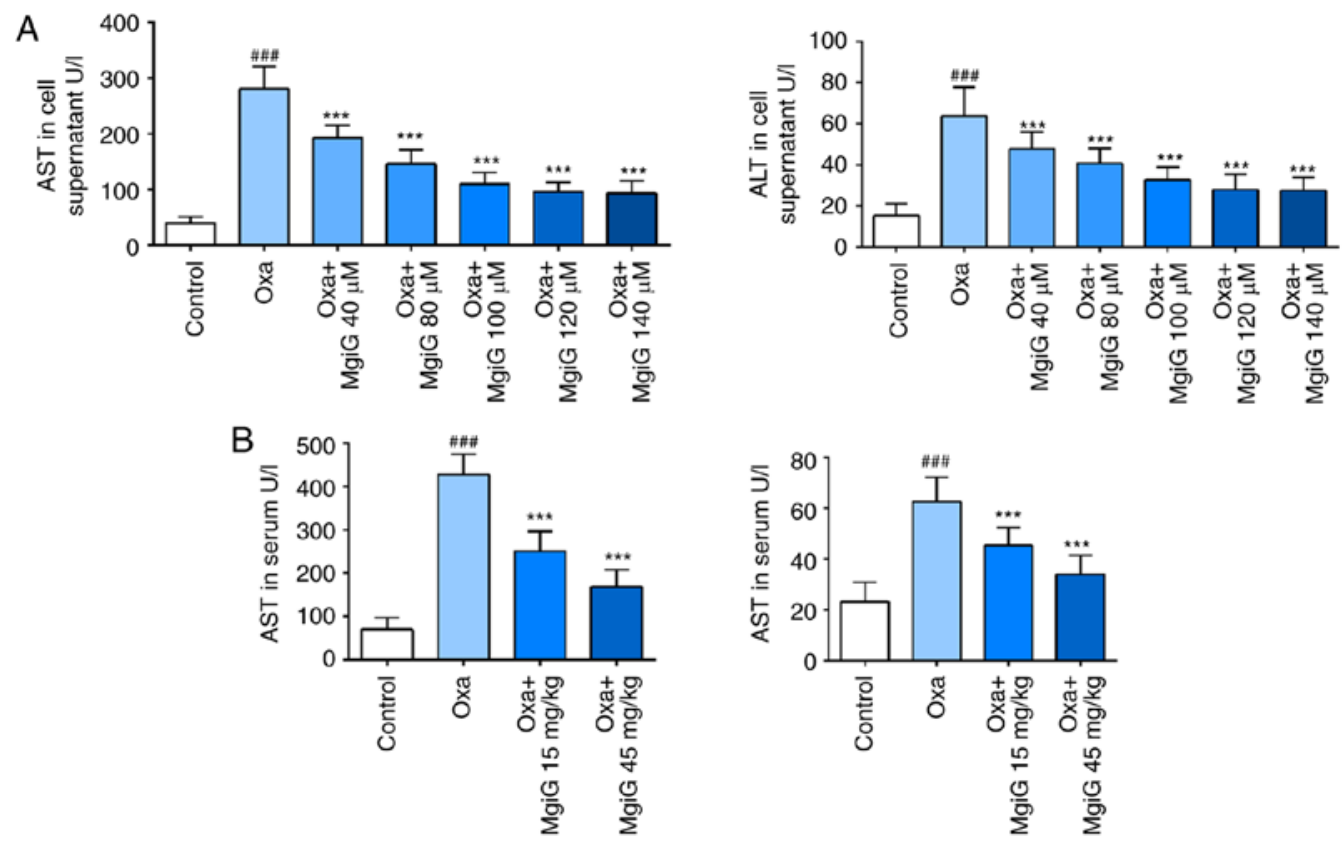

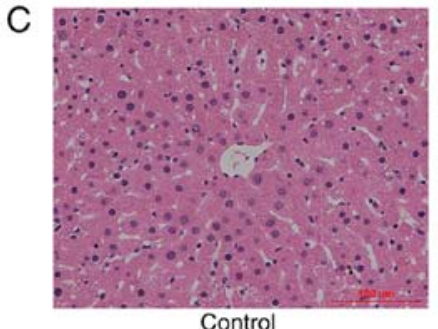

Control

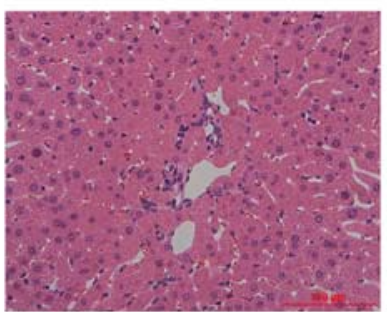

Oxa+MgiG 15 mg/kg

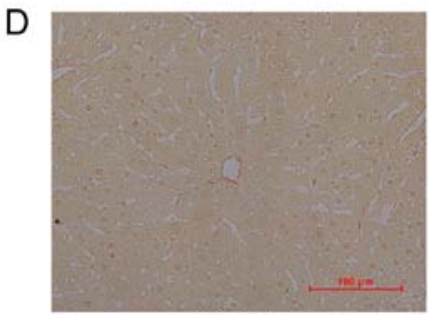

Control

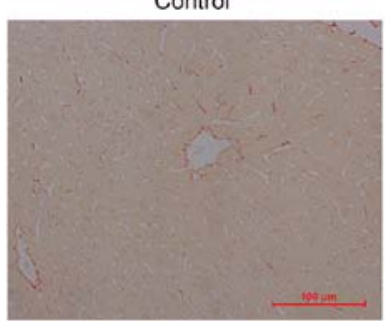

Oxa+MgiG 15 mg/kg

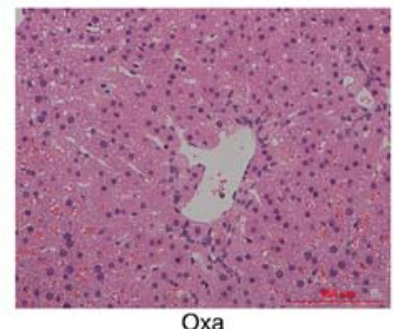

Oxa

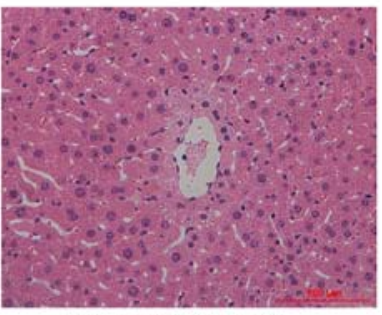

Oxa+MgiG 45 mg/kg

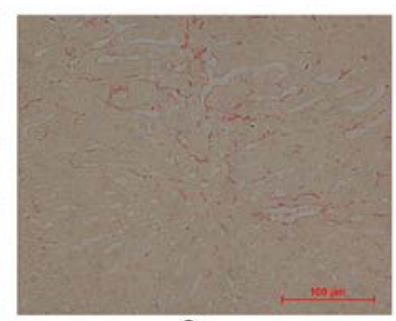

Oxa

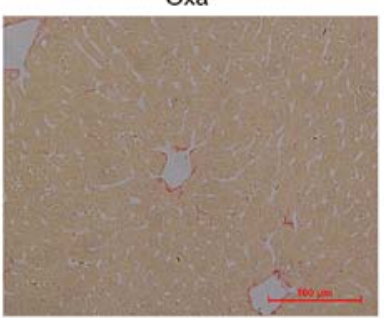

Oxa+MgiG 45 mg/kg
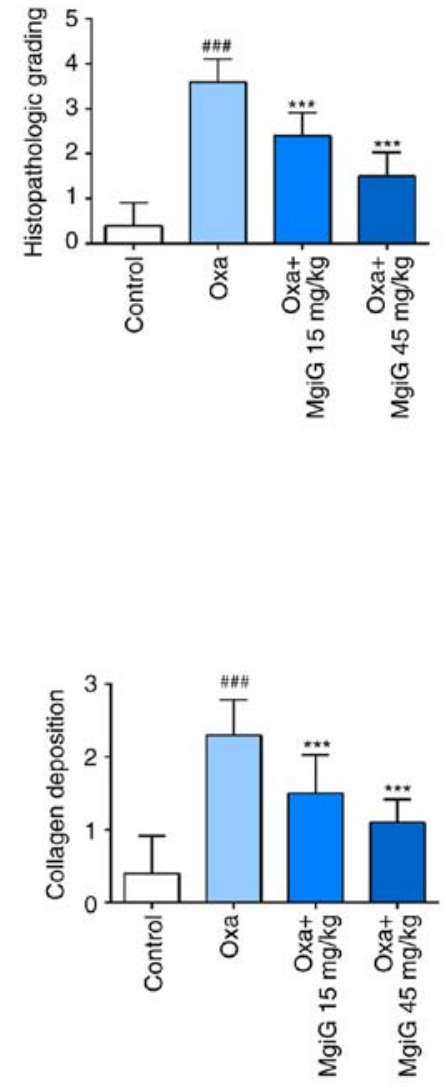

Figure 1. Effects of MgiG on aminotransferases in serum and cell supernatants and histopathological alterations in hepatic tissues induced by oxaliplatin. (A) Levels of AST and ALT in cell supernatants following treatment of LO2 cells with oxaliplatin and MgiG. (B) Levels of AST and ALT in serum from mice treated with oxaliplatin and MgiG. (C) Representative images from hematoxylin and eosin staining of liver tissue sections from each experimental group, and scoring results for histopathologic grading. (D) Representative images from Sirius red staining of liver tissue sections and scoring results for collagen deposition. Data are presented as mean \pm standard deviation $\left(\mathrm{n}=10\right.$ mice per group; $\mathrm{n}=3$ for in vitro results). ${ }^{\# \# \#} \mathrm{P}<0.001$ compared with control; ${ }^{* * *} \mathrm{P}<0.001$ compared with oxaliplatin alone. MgiG, magnesium isoglycyrrhizinate; AST, aspartate aminotransferase; ALT, aminotransferase; Oxa, oxaliplatin. 
A
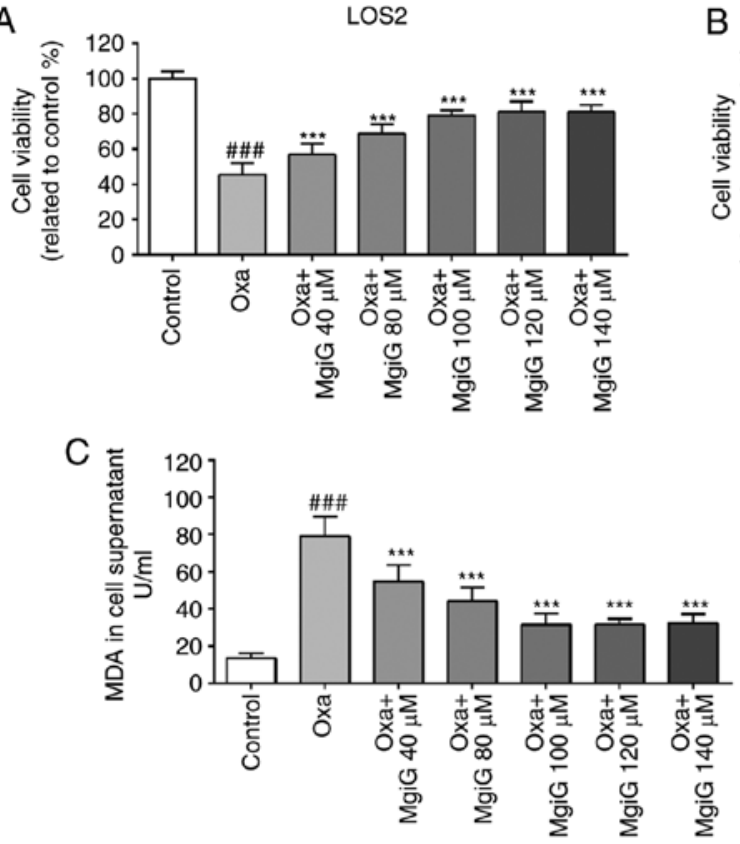

$\mathrm{E}$

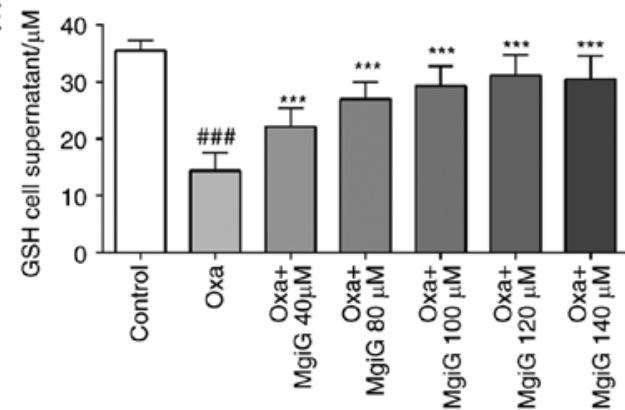

G

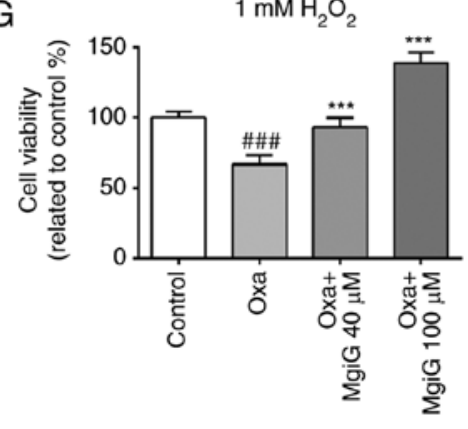

B HT-29
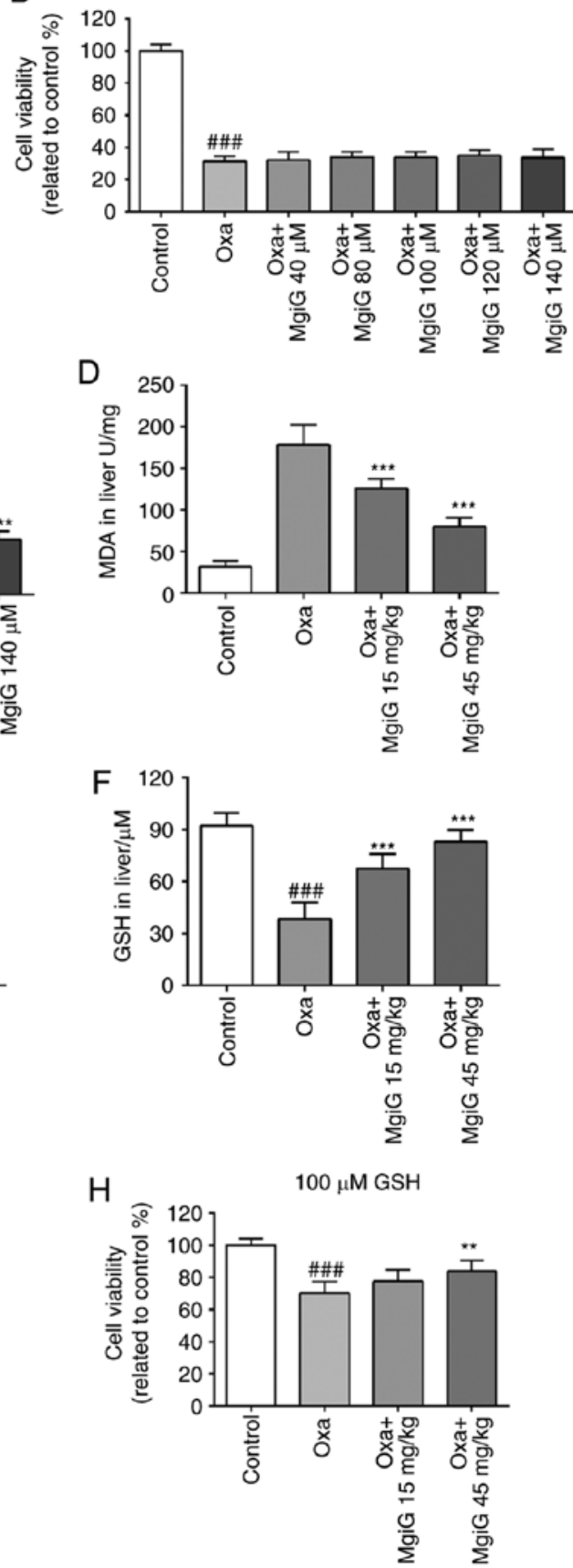

Figure 2. MgiG improves cell viability following oxaliplatin exposure and protects liver cells by attenuating oxaliplatin-induced oxidative stress. (A) Viability of LO2 cells and of (B) HT-29 cells following oxaliplatin and MgiG treatment. (C) MDA levels in cells supernatants. (D) MDA levels in livers from experimental mice. (E) GSH levels in cells supernatants. (F) GSH levels in livers from experimental mice. (G) Cell viability of LO2 cells following oxaliplatin and MgiG treatment with the presence of supplementary $1 \mathrm{mM} \mathrm{H}_{2} \mathrm{O}_{2}$, or $(\mathrm{H}) 100 \mu \mathrm{M} \mathrm{GSH}$ in the medium. Data are presented as mean \pm standard deviation ( $\mathrm{n}=10$ mice per group; $\mathrm{n}=3$ for in vitro results). ${ }^{\# \# *} \mathrm{P}<0.001$ compared with control; ${ }^{* *} \mathrm{P}<0.01$ and ${ }^{* * *} \mathrm{P}<0.001$ compared with oxaliplatin alone. MgiG, magnesium isoglycyrrhizinate; MDA, malondialdehyde; GSH, glutathione; Oxa, oxaliplatin.

improved viability by $109,38 \%$ higher than the control group (Fig. 2G). In the viability assay with supplementary GSH, oxaliplatin challenge attenuated cell viability by $30 \%$. The cell viability in MgiG-treated groups also increased (Fig. 2H). High-dose MgiG treatment improved cell viability by $20 \%$, and there was no significant difference between the viabilities of the oxaliplatin-treated group and the low-dose MgiG group (Fig. 2H).

Next, the expression of genes classically associated with anti- and pro-oxidative functions was investigated (27). The expression levels of vital genes implicated in the response to oxidative stress, Mt1, PRDX1 and SOD2, were measured in the liver of oxaliplatin-treated mice by RT-qPCR and western blotting. The results demonstrated that both mRNA and protein expression levels of these genes were increased in the livers of oxaliplatin-treated mice compared with control mice (Fig. 3). MgiG treatment, however, effectively attenuated the increased expression of these genes (Fig. 3). In addition, all three indicators were more improved with high-dose MgiG than with low-dose MgiG (Fig. 3). 

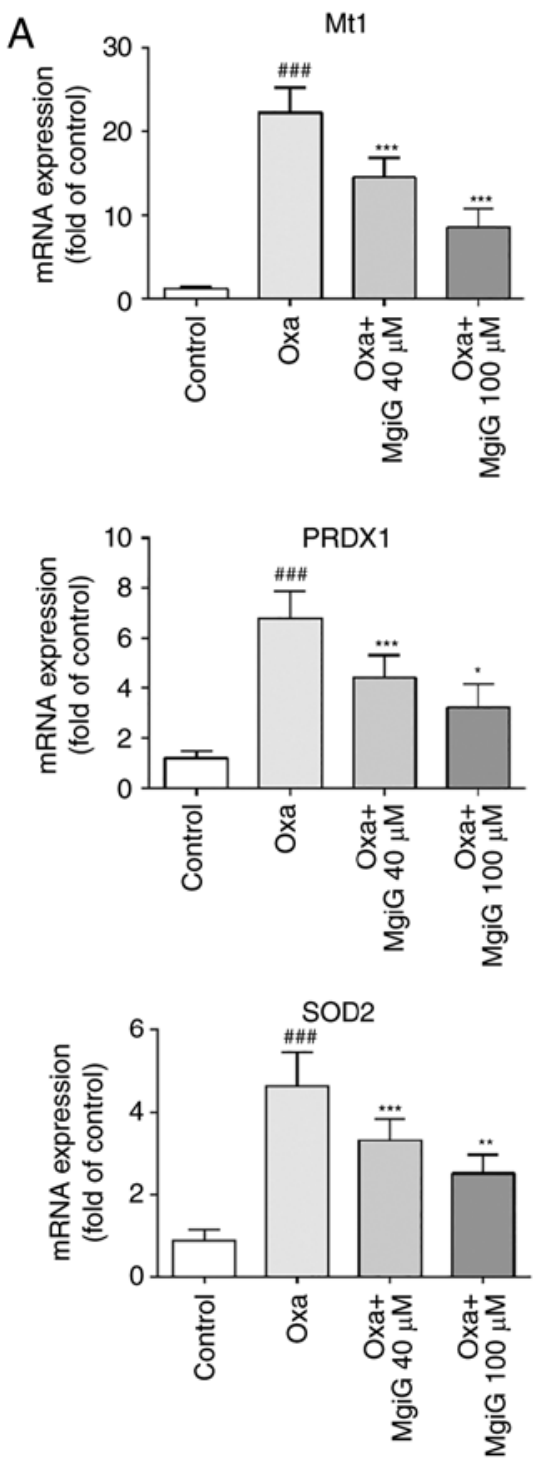

B
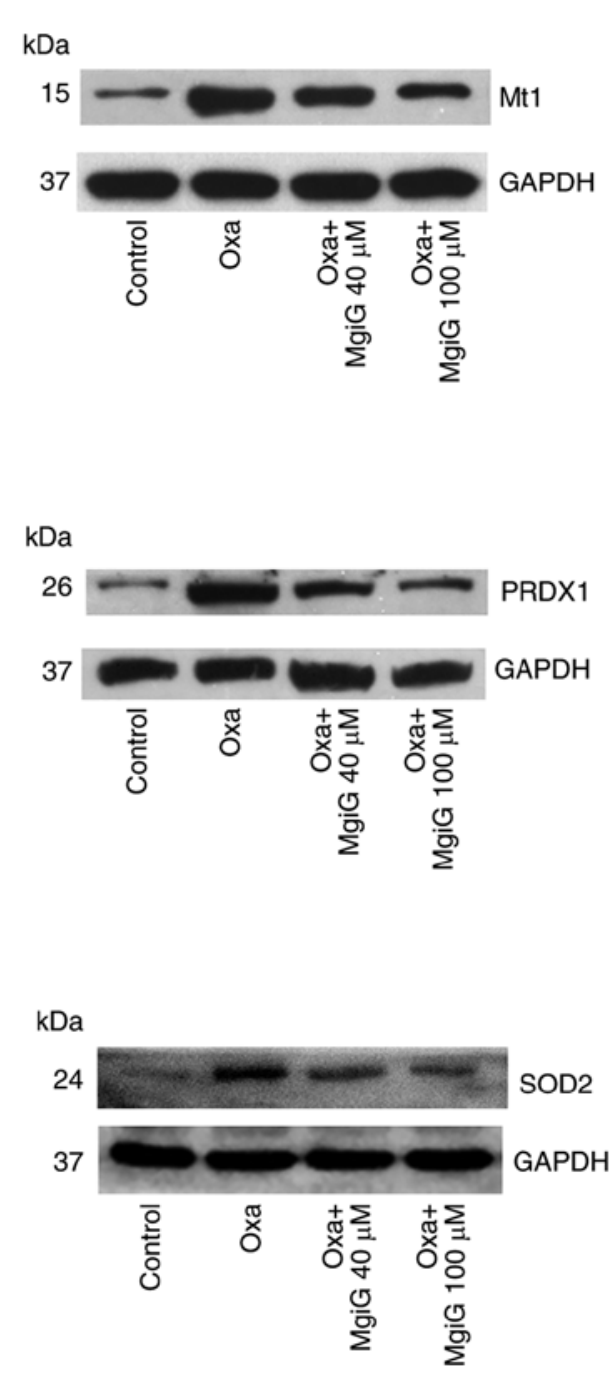
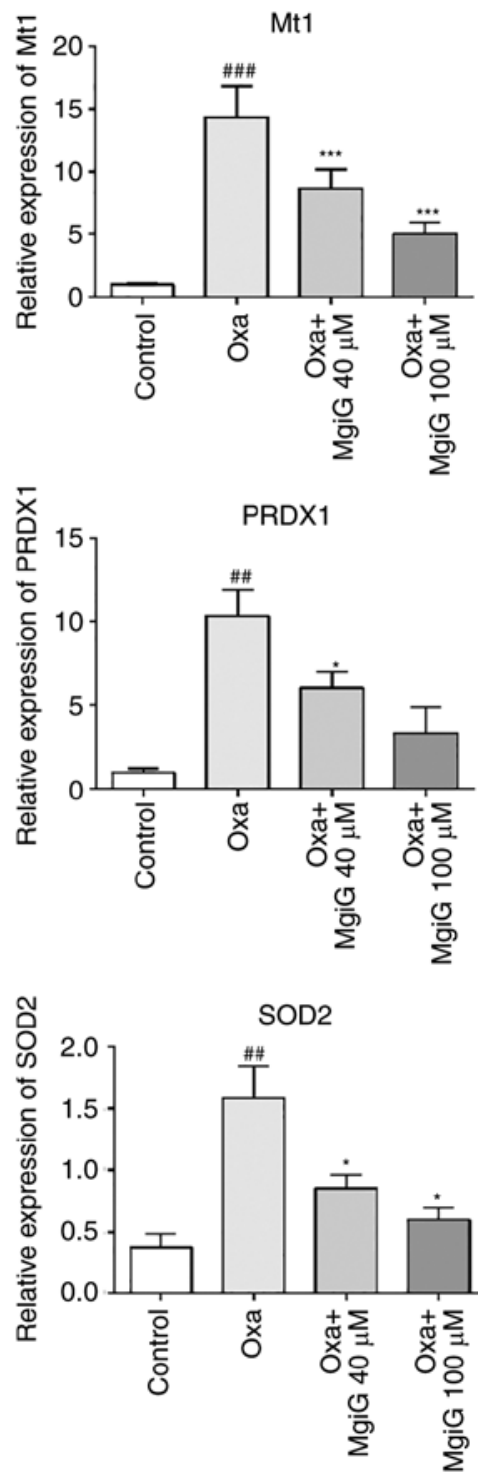

Figure 3. MgiG regulates the expression of genes in the oxidative stress pathway. (A) mRNA expression levels of Mt1, PRDX1 and SOD2. (B) Protein expression levels of Mt1, PRDX1 and SOD2, measured by western blotting. Representative blots and quantification is shown. Results were normalized to GAPDH. Data are presented as mean \pm standard deviation ( $\mathrm{n}=10$ mice per group; $\mathrm{n}=3$ for in vitro results). ${ }^{\# \#} \mathrm{P}<0.01$ and ${ }^{\# \#} \mathrm{P}<0.001$ compared with control; ${ }^{*} \mathrm{P}<0.05$, ${ }^{* *} \mathrm{P}<0.01$ and ${ }^{* * *} \mathrm{P}<0.001$ compared with oxaliplatin alone. MgiG, magnesium isoglycyrrhizinate; Mt1, metallothionein 1; PRDX1, peroxiredoxin 1; SOD2, superoxide dismutase 2; Oxa, oxaliplatin.

MgiG inhibits IL-6 pathway activation induced by oxaliplatin. Previous studied have reported that the IL-6 pathway is a core signaling pathway activated during oxaliplatin-related liver damage $(11,21)$. IL-6 levels in liver homogenates were measured by ELISA and RT-qPCR. The secreted levels and mRNA expression of IL-6 in liver homogenates were increased following oxaliplatin administration and this effect was significantly attenuated by MgiG treatment (Fig. 4A and B). The levels of IL-6 in the high-dose group were lower compared with the low-dose group. STAT3 is one of the most important transcription factors downstream of IL-6. Its expression in liver homogenates was also tested by RT-qPCR and Western blot. The RT-qPCR results demonstrated that STAT3 expression was increased by 3.4 -fold in the oxaliplatin-administered group, and that this increase was reversed by MgiG treatment (Fig. 4C). The western blot analysis demonstrated similar results (Fig. 4D and E).
MgiG reverses the pro-thrombotic environment within oxaliplatin-injured hepatic sinusoids. In most previous studies of oxaliplatin-induced liver injury, activation of the coagulation system was one of the most-discussed mechanisms $(11,12,21,26,31)$. In the present study, ELISA results revealed that levels of $\mathrm{vWF}$, a key component in platelet adhesion, were significantly increased in the livers of animals administered with oxaliplatin (Fig. 4F). MgiG treatment significantly attenuated this increase (Fig. 4F). This effect was also evident at the mRNA and protein level, as measured by RT-qPCR and western blotting (Fig. 4G-I). Expression of PAI-1 (also known as SERPINE1) in the livers of experimental animals was also increased as a result of oxaliplatin exposure, as measured by RT-qPCR (Fig. 4J). MgiG treatment notably inhibited this increase (Fig. 4J). Western blott analysis revealed similar results at the protein level (Fig. 4K and L). In both experiments, high-dose MgiG 
A
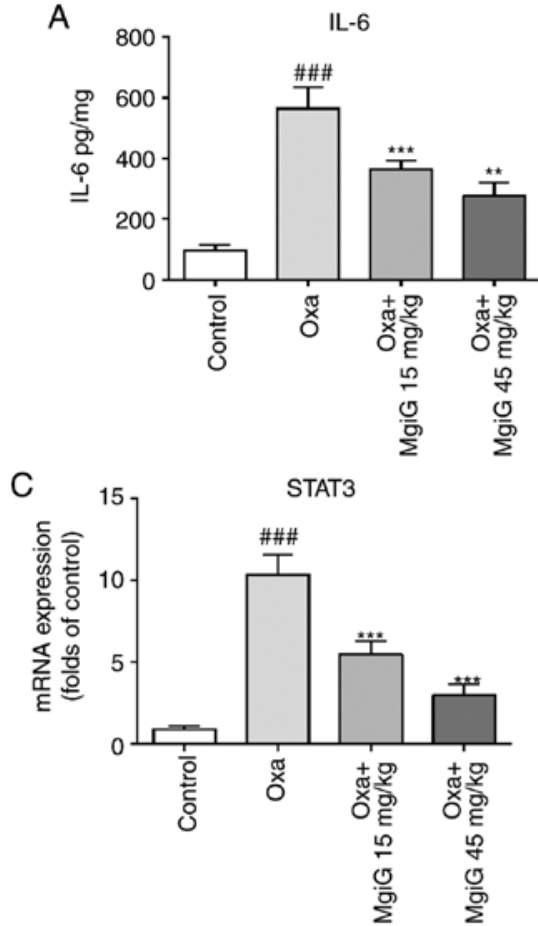

$\mathrm{E}$

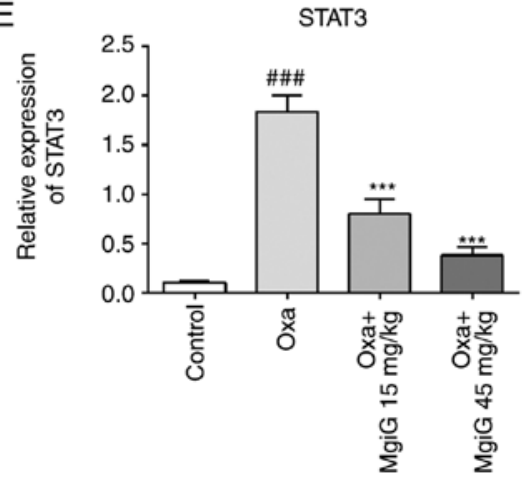

B

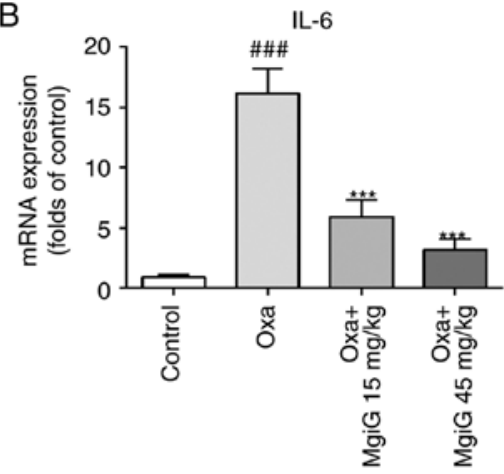

D $\mathrm{kDa}$

80

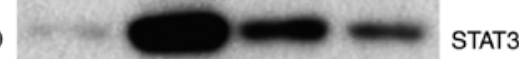

37

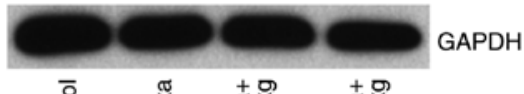

$\mathrm{F}$

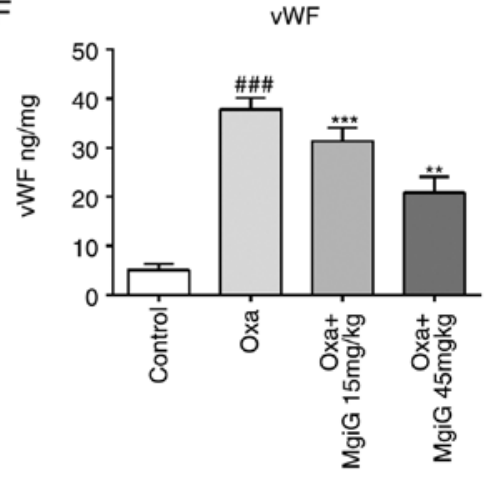

$\mathrm{G}$
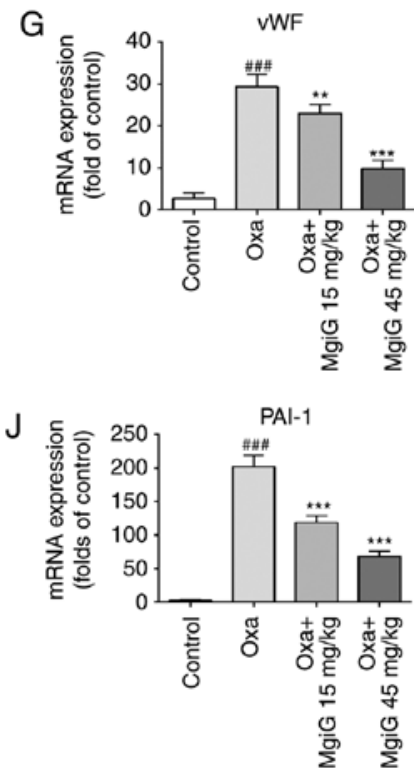

$\mathrm{H}$ kDa
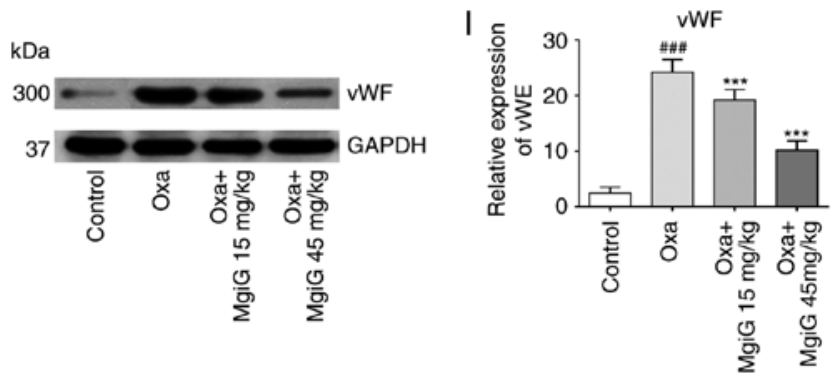

$\mathrm{K} \mathrm{kDa}$

48

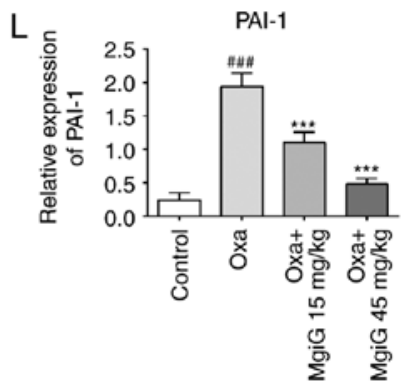

Figure 4. MgiG inhibits oxaliplatin-induced activation of the IL-6 pathway and the coagulation cascade. (A) Secreted levels and (B) mRNA expression levels of IL-6. (C) mRNA expression levels, and (D and E) protein expression levels of STAT3. (F) Secreted levels, (G) mRNA expression levels and (H and I) protein expression levels of vWF. (J) mRNA expression levels and (K and L) protein expression levels of PAI-1. Western blot results were normalized to GAPDH. Data are presented as mean \pm standard deviation ( $\mathrm{n}=10$ mice per group; $\mathrm{n}=3$ for in vitro results). ${ }^{\# \# \#} \mathrm{P}<0.001$ compared with control; ${ }^{* * *} \mathrm{P}<0.01$ and ${ }^{* * * *} \mathrm{P}<0.001$ compared with oxaliplatin alone. MgiG, magnesium isoglycyrrhizinate; IL-6, interleukin-6; STAT3, signal transducer and activator of transcription 3; vWF, von Willebrand factor; PAI-1, plasminogen activator inhibitor-1; Oxa, oxaliplatin. 
had an improved protective effect compared with low-dose MgiG.

\section{Discussion}

Oxaliplatin-induced liver injury is a primary limiting factor affecting the efficacy of oxaliplatin-based chemotherapy in patients with CRLM (7). Medications that can protect liver cells against damage caused by oxaliplatin are urgently needed. However, relevant studies are very rare. There is only one known study regarding the use of reduced glutathione in oxaliplatin-induced acute liver injury in vivo (19). To the best of our knowledge, the present study is the first report of a medication to treat or prevent oxaliplatin-caused liver injury using both in vivo and in vitro experiments. The current study presented novel insights into the mechanisms of oxaliplatin-induced liver injury and might represent a potential strategy for its treatment in the future.

It has been demonstrated that $\mathrm{MgiG}$ has an improved liver-protective effect compared with either glycyrrhizin or $\beta$-glycyrrhizic acid in carbon tetrachloride- and D-galactosamine-induced liver damage animal models. Previous studies have also demonstrated that MgiG attenuates liver injury caused by toxins and other factors, such as free fatty acids, ethanol and ischemia/reperfusion (13-15,20,32). MgiG has already been clinically used as a drug in the treatment of hepatitis and toxin-associated liver injury. In addition, a previous study has reported the hepatoprotective effect of MgiG in chemotherapy reagent-induced liver injury caused by cyclophosphamide (18).

In most previous studies, chemotherapy-associated liver injury animal models were created by administration of toxic reagents (33-35), such as tetrachloromethane, D-galactosamine and monocrotaline. However, there was a great risk that the underlying molecular mechanisms leading to the pathological changes in the liver of experimental animals would be different from those of patients receiving chemotherapy, because of the different drugs used to cause it. The side-effects induced by toxic agents are also a difficulty, including the renal toxicity of tetrachloromethane and pulmonary hypertension as a result of monocrotaline exposure $(36,37)$. More valuable information could be derived from studying the liver-protecting effect against chemotherapy in an animal model induced by a chemotherapy reagent, rather than toxins. The liver injury animal model in the present study was established by intraperitoneal injection of oxaliplatin, and the drug was administered to the animals based on a treatment schedule comparable with that of human patients receiving chemotherapy in the clinic. In this way, the aim of the present study was to more accurately mimic the pathogenic process of oxaliplatin-associated liver injury.

AST and ALT are the most reliable biochemical markers of liver damage. The present results revealed significant increases in transaminase levels both in serum and in LO2 cell supernatants following oxaliplatin exposure. Grading results from $H \& E$ and Sirius red staining demonstrated pathological alterations within the livers of experimental animals, including vast areas of cellular necrosis, extensive vacuolization, inflammatory cell infiltration and fibrosis. The experimental findings indicated the occurrence of severe liver injury caused by oxaliplatin. MgiG treatment significantly ameliorated the injury, accompanied by an improvement in transaminase levels both in vivo and in vitro and reversion of the pathological changes.

Potential limitations of the current study may be that MgiG served its protective role by directly inactivating the chemotherapy drug or by protecting other types of cells, such as the cancer cells, from oxaliplatin. While the cell viability assay revealed a substantial protective effect of MgiG in LO2 cells, there was no significant difference in Ht-29 cell viability with or without MgiG treatment, suggesting that MgiG specifically protected hepatic cells from oxaliplatin without attenuating its efficacy on colon cancer cells. This is a very interesting phenomenon, and several reasons might explain it. Firstly, liver is the primary source of GSH production in the human body. There are studies indicating that GSH is closely associated with drug inactivation, and GSH is elevated in a number of drug-resistant tumor cell lines (38-40). In addition, alleviated oxidative stress is one of the mechanisms by which oxaliplatin functions. MgiG might, by an unknown mechanism, promote the synthesis of GSH by liver cells. Other explanations can be considered; a previous study has demonstrated that glycyrrhetinic acid (GA) administration increased the expression and activity of UDP-glucuronyltransferases (UGTs) in the liver of experimental animals while simultaneously attenuating UGT activity in the intestine (41). UGTs are known as the primary enzymatic family affecting the pharmacokinetic and pharmacodynamic properties of xenobiotics. Theoretically, MgiG is also a derivative of glycyrrhizin, as is GA, suggesting that they might share some metabolic process in the body. Therefore, increasing UGT activity in the liver might also be a potential mechanism by which MgiG attenuated oxaliplatin-induced liver injury.

Previous studies indicated that increased oxidative stress is an important cause of oxaliplatin-induced hepatotoxicity $(11,21,25)$. As the end-product of lipid breakdown, MDA is recognized as a reliable indicator sensitive to oxidative stress in hepatic lesions (42). The present study demonstrated that MgiG eliminated the changes in oxidative stress indicators (GSH and MDA) caused by oxaliplatin exposure. When additional oxidative stress was present, the hepatic toxicity of oxaliplatin was partially abolished, and MgiG restored cell viability to even higher levels than those of the control group. With the presence of extra reductant, the hepatic toxicity of oxaliplatin was also partially abolished, and the protective effect of MgiG decreased simultaneously. Taken together, these data suggested that oxaliplatin caused liver injury by increasing oxidative stress, and MgiG protected liver cells by attenuating oxidative stress. The increased expression of a variety of genes implicated in responses to oxidative stress (Mt1,PRDX1 and SOD2) was also diminished following MgiG treatment at both the transcriptional and the translational level.

A broad spectrum of studies suggested that oxaliplatin induced inflammatory responses in the liver. Oxaliplatin has been reported to cause overproduction of various pro-inflammation cytokines, including CXC motif chemokine ligand 1 (CXCL1; also known as IL-8), monocyte chemoattractant protein-1 (MCP-1) and IL-6 (11,21). Although inflammation is unlikely to be the main initiator of oxaliplatin-induced liver 
injury, the IL-6 pathway might become activated in response to toxic damage or ischemic injury (43). As a vital transcription factor downstream of IL-6, STAT3 mediates the function of anti-apoptosis, reducing oxidative stress and maintaining capillary integrity. The present study demonstrated that oxaliplatin stimulation increased IL-6 and STAT3 expression, while this effect was significantly antagonized by MgiG, confirming the anti-inflammatory role of MgiG in oxaliplatin-induced hepatic injury.

Coagulation pathways have been implicated in the pathogenic process of oxaliplatin-induced liver injury $(11,12,21)$. More support for this hypothesis came from the observation that patients taking aspirin tended to be at lower risk of developing oxaliplatin-induced SOS (44). A possible explanation regarding how a prothrombotic environment contributes to the development of SOS involves the generation of microthrombi within hepatic sinusoids that subsequently become occluded, exacerbating the elevated oxidative stress. Both vWF and PAI-1 are principle markers of the coagulation cascade. The present results demonstrated that vWF and PAI-1 were overexpressed following oxaliplatin exposure, and $\mathrm{MgiG}$ treatment effectively restored the levels of these factors. The changes in PAI-1 mRNA levels measured by RT-qPCR were much higher than the changes in protein levels measured by western blot analysis. PAI-1 is a primary inhibitor of the plasminogen activators. The activity of PAI-1 is regulated by many factors, including circadian rhythm, blood glucose levels, insulin and insulin-like molecules, and even its own genetic variation at polymorphic loci is associated with differences in plasma PAI-1 levels (45-50). Therefore, there might be other regulatory mechanisms occurring following the transcription of the protein, leading to smaller changes in protein levels compared with mRNA levels. In addition, the hepatocyte is the main source of PAI-1 synthesis in the body, and the produced protein is then secreted into the serum (51). This process could also result in smaller changes in liver PAI-1 protein levels compared with mRNA levels.

There are some limitations in the present study. The phenomenon of $\mathrm{MgiG}$ specifically protecting hepatocytes but having no influence on the effectiveness of oxaliplatin in colon cancer cells is very interesting and requires further investigation. Deeper exploration of the detailed mechanism by which MgiG functions is needed, particularly the metabolic processes of $\mathrm{MiG}$ in the body. Our group will continue this line of research in future studies.

In summary, the present study demonstrated that MgiG effectively attenuated oxaliplatin-induced liver injury in vitro and in vivo. The protective effect of $\mathrm{MgiG}$ on oxaliplatin-induced liver injury was correlated with the attenuation of oxidative stress, the IL- 6 pathway and the coagulation pathway. The present findings revealed that MgiG may have potential value as a treatment or prevention agent against oxaliplatin-induced liver injury in clinical practice.

\section{Acknowledgements}

We are grateful to $\mathrm{Dr} \mathrm{Su}$ and his colleague from the Department of Pathology, Qilu Hospital for the assistance for the construction and analysis of tissue slices.

\section{Funding}

This study was supported by the National Natural Science Foundation of China (grant no. 81572414) and the Natural Science Foundation of Shandong Province (grant no. ZR2018BH023).

\section{Availability of data and materials}

The analyzed datasets generated during the study are available from the corresponding author on reasonable request.

\section{Authors' contributions}

JN was responsible for designing of the study and critical review of manuscript. XZ was responsible for designing and performing the study, literature research and manuscript drafting. YW participated in the animal model establishment, cell culturing and statistical analysis. BW and CP participated in the design of the study and sequence alignment. ZN and ZL participated in sequence alignment and immunoassays. All authors read and approved the final manuscript.

\section{Ethics approval and consent to participate}

The present research was approved by the Institutional Animal Care and Use Committee of Shandong University (Jinan, China).

\section{Patient consent for publication}

Not applicable.

\section{Competing interests}

The authors declare that they have no competing interests.

\section{References}

1. Torre LA, Bray F, Siegel RL, Ferlay J, Lortet-Tieulent J and Jemal A: Global cancer statistics, 2012. CA Cancer J Clin 65: 87-108, 2015.

2. Maughan TS, Adams RA, Smith CG, Meade AM, Seymour MT, Wilson RH, Idziaszczyk S, Harris R, Fisher D, Kenny SL, et al: Addition of cetuximab to oxaliplatin-based first-line combination chemotherapy for treatment of advanced colorectal cancer: Results of the randomised phase 3 MRC COIN trial. Lancet 377: 2103-2114, 2011.

3. Beppu T, Sakamoto Y, Hayashi H and Baba H: Perioperative chemotherapy and hepatic resection for resectable colorectal liver metastases. Hepatobiliary Surg Nutr 4: 72-75, 2015.

4. André T, Boni C, Mounedji-Boudiaf L, Navarro M, Tabernero J, Hickish T, Topham C, Zaninelli M, Clingan P, Bridgewater J, et al: Oxaliplatin, fluorouracil, and leucovorin as adjuvant treatment for colon cancer. N Engl J Med 350: 2343-2351, 2004.

5. André T, Boni C, Navarro M, Tabernero J, Hickish T, Topham C, Bonetti A, Clingan P, Bridgewater J, Rivera F and de Gramont A: Improved overall survival with oxaliplatin, fluorouracil, and leucovorin as adjuvant treatment in stage II or III colon cancer in the MOSAIC trial. J Clin Oncol 27: 3109-3116, 2009.

6. Portier G, Elias D, Bouche O, Rougier P, Bosset JF, Saric J, Belghiti J, Piedbois P, Guimbaud R, Nordlinger B, et al: Multicenter randomized trial of adjuvant fluorouracil and folinic acid compared with surgery alone after resection of colorectal liver metastases: FFCD ACHBTH AURC 9002 trial. J Clin Oncol 24: 4976-4982, 2006. 
7. Rubbia-Brandt L, Lauwers GY, Wang H, Majno PE, Tanabe K, Zhu AX, Brezault C, Soubrane O, Abdalla EK, Vauthey JN, et al: Sinusoidal obstruction syndrome and nodular regenerative hyperplasia are frequent oxaliplatin-associated liver lesions and partially prevented by bevacizumab in patients with hepatic colorectal metastasis. Histopathology 56: 430-439, 2010.

8. Slade JH, Alattar ML, Fogelman DR, Overman MJ, Agarwal A, Maru DM, Coulson RL, Charnsangavej C, Vauthey JN, Wolff RA and Kopetz S: Portal hypertension associated with oxaliplatin administration: Clinical manifestations of hepatic sinusoidal injury. Clin Colorectal Cancer 8: 225-230, 2009.

9. Hubert C, Fervaille C, Sempoux C, Horsmans Y, Humblet Y Machiels JP, Zech F, Ceratti A and Gigot JF: Prevalence and clinical relevance of pathological hepatic changes occurring after neoadjuvant chemotherapy for colorectal liver metastases. Surgery 147: 185-194, 2010.

10. Robinson SM, Wilson CH, Burt AD, Manas DM and White SA: Chemotherapy-associated liver injury in patients with colorectal liver metastases: A systematic review and meta-analysis. Ann Surg Oncol 19: 4287-4299, 2012.

11. Rubbia-Brandt L, Tauzin S, Brezault C, Delucinge-Vivier C, Descombes P, Dousset B, Majno PE, Mentha G and Terris B: Gene expression profiling provides insights into pathways of oxaliplatin-related sinusoidal obstruction syndrome in humans. Mol Cancer Ther 10: 687-696, 2011.

12. Agostini J, Benoist S, Seman M, Julié C, Imbeaud S, Letourneur F Cagnard N, Rougier P, Brouquet A, Zucman-Rossi J and Laurent-Puig P: Identification of molecular pathways involved in oxaliplatin-associated sinusoidal dilatation. J Hepatol 56: 869-876, 2012

13. Sun L, Shen J, Pang X, Lu L, Mao Y and Zeng M: Phase I safety and pharmacokinetic study of magnesium isoglycyrrhizinate after single and multiple intravenous doses in chinese healthy volunteers. J Clin Pharmacol 47: 767-773, 2007.

14. Xu Q, Wang J, Chen F, Lin K, Zhu M, Chen L, Zhou X, Li C and $\mathrm{Zhu} \mathrm{H}$ : Protective role of magnesium isoglycyrrhizinate in non-alcoholic fatty liver disease and the associated molecular mechanisms. Int J Mol Med 38: 275-282, 2016.

15. Cheng Y, Zhang J, Shang J and Zhang L: Prevention of free fatty acid-induced hepatic lipotoxicity in HepG2 cells by magnesium isoglycyrrhizinate in vitro. Pharmacology 84: 183-190, 2009.

16. Zhao HJ, Xiao L, Glizila B, Zhang H, Mao R, Xiong Y, Xu L, Shu MY, Bai YW and Bao YX: The effect and comparison of commonly used liver-protection drugs for irradiated HL-7702 by X. Zhonghua Gan Zang Bing Za Zhi 25: 612-617, 2017 (In Chinese).

17. Wang Y,Zhang Z, Wang X, Qi D, Qu A and Wang G: Amelioration of ethanol-induced hepatitis by magnesium isoglycyrrhizinate through inhibition of neutrophil cell infiltration and oxidative damage. Mediators Inflamm 2017: 3526903, 2017.

18. Jiang W, Liu J, Li P, Lu Q, Pei X, Sun Y, Wang G and Hao K: Magnesium isoglycyrrhizinate shows hepatoprotective effects in a cyclophosphamide-induced model of hepatic injury. Oncotarget 8: 33252-33264, 2017.

19. Lin Y, Li Y, Hu X, Liu Z, Chen J, Lu Y, Liu J, Liao S, Zhang Y, Liang $\mathrm{R}$, et al: The hepatoprotective role of reduced glutathione and its underlying mechanism in oxaliplatin-induced acute liver injury. Oncol Lett 15: 2266-2272, 2018.

20. He Y,Zeng F, Liu Q, Ju W, Fu H, Hao H, Li L and Xie Y: Protective effect of magnesium isoglycyrrhizinate on ethanol-induced testicular injuries in mice. J Biomed Res 24: 153-160, 2010.

21. Robinson SM, Mann J, Vasilaki A, Mathers J, Burt AD, Oakley F, White SA and Mann DA: Pathogenesis of FOLFOX induced sinusoidal obstruction syndrome in a murine chemotherapy model. J Hepatol 59: 318-326, 2013.

22. Xiong W, Ren ZG, Qiu SJ, Sun HC, Wang L, Liu BB, Li QS, Zhang W, Zhu XD, Liu L, et al: Residual hepatocellular carcinoma after oxaliplatin treatment has increased metastatic potential in a nude mouse model and is attenuated by Songyou Yin. BMC Cancer 10: 219, 2010

23. Kelley MR, Jiang Y, Guo C, Reed A, Meng H and Vasko MR Role of the DNA base excision repair protein, APE1 in cisplatin, oxaliplatin, or carboplatin induced sensory neuropathy. PLoS One 9: e106485, 2014

24. Livak KJ and Schmittgen TD: Analysis of relative gene expression data using real-time quantitative PCR and the 2(-Delta Delta C(T)) method. Methods 25: 402-408, 2001.

25. Kweekel DM, Gelderblom H and Guchelaar HJ: Pharmacology of oxaliplatin and the use of pharmacogenomics to individualize therapy. Cancer Treat Rev 31: 90-105, 2005.
26. McWhirter D, Kitteringham N, Jones RP, Malik H, Park K and Palmer D: Chemotherapy induced hepatotoxicity in metastatic colorectal cancer: A review of mechanisms and outcomes. Crit Rev Oncol Hematol 88: 404-415, 2013.

27. Hua W, Huang HZ, Tan LT, Wan JM, Gui HB, Zhao L, Ruan XZ, Chen XM and Du XG: CD36 mediated fatty acid-induced podocyte apoptosis via oxidative stress. PLoS One 10: e0127507, 2015.

28. Zhang S, Li X, Jourd'heuil FL, Qu S, Devejian N, Bennett E, Jourd'heuil D and Cai C: Cytoglobin promotes cardiac progenitor cell survival against oxidative stress via the upregulation of the $\mathrm{NF} \kappa \mathrm{B} / \mathrm{iNOS}$ signal pathway and nitric oxide production. Sci Rep 7: 10754, 2017.

29. Arafa E, Bondzie PA, Rezazadeh K, Meyer RD, Hartsough E, Henderson JM, Schwartz JH, Chitalia V and Rahimi N: TMIGD1 is a novel adhesion molecule that protects epithelial cells from oxidative cell injury. Am J Pathol 185: 2757-2767, 2015.

30. Madesh M and Hajnóczky G: VDAC-dependent permeabilization of the outer mitochondrial membrane by superoxide induces rapid and massive cytochrome c release. J Cell Biol 155: 1003-1015, 2001

31. Lu Z, Cheng D, Yin J, Wu R, Zhang G, Zhao Q, Wang N, Wang F and Liang M: Antithrombin III protects against contrast-induced nephropathy. EBioMedicine 17: 101-107, 2017.

32. Gu X, Dong L, Liu Y and Yu F: Protective effect of magnesium isoglycyrrhizinate on acute hepatic injury induced by $\mathrm{CCl} 4$ in mice. China Pharmacist 12: 37-39, 2009.

33. DeLeve LD, McCuskey RS, Wang X, Hu L, McCuskey MK, Epstein RB and Kanel GC: Characterization of a reproducible rat model of hepatic veno-occlusive disease. Hepatology 29: 1779-1791, 1999.

34. Ma JQ, Ding J, Zhao H and Liu CM: Puerarin attenuates carbon tetrachloride-induced liver oxidative stress and hyperlipidaemia in mouse by JNK/c-Jun/CYP7A1 pathway. Basic Clin Pharmacol Toxicol 115: 389-395, 2014.

35. Nakamura K, Hatano E, Miyagawa-Hayashino A, Okuno M, Koyama Y, Narita M, Seo S, Taura K and Uemoto S: Soluble thrombomodulin attenuates sinusoidal obstruction syndrome in rat through suppression of high mobility group box 1 . Liver Int 34: 1473-1487, 2014

36. Schultze AE and Roth RA: Chronic pulmonary hypertension-the monocrotaline model and involvement of the hemostatic system. J Toxicol Environ Health B Crit Rev 1: 271-346, 1998.

37. Hassan MH, Bahashawan SA, Abdelghany TM, Abd-Allah GM and Ghobara MM: Crocin abrogates carbon tetrachloride-induced renal toxicity in rats via modulation of metabolizing enzymes and diminution of oxidative stress, apoptosis, and inflammatory cytokines. J Biochem Mol Toxicol 29: 330-339, 2015.

38. Bray TM and Taylor CG: Tissue glutathione, nutrition, and oxidative stress. Can J Physiol Pharmacol 71: 746-751, 1993.

39. Lu SC: Regulation of hepatic glutathione synthesis: Current concepts and controversies. FASEB J 13: 1169-1183, 1999.

40. Mulcahy RT, Bailey HH and Gipp JJ: Up-regulation of gamma-glutamylcysteine synthetase activity in melphalan-resistant human multiple myeloma cells expressing increased glutathione levels. Cancer Chemother Pharmacol 34: 67-71, 1994.

41. Li FY, Xie H, Weng L, Wang H, Cao LJ, Hao HP and Wang GJ: Effects of diammonium glycyrrhizinate on hepatic and intestinal UDP-Glucuronosyltransferases in rats: Implication in herb-drug interactions. Chin J Nat Med 14: 534-540, 2016.

42. Liu M, Xu H, Zhang L, Zhang C, Yang L, Ma E, Liu L and Li Y: Salvianolic acid B inhibits myofibroblast transdifferentiation in experimental pulmonary fibrosis via the up-regulation of $\mathrm{Nrf2}$. Biochem Biophys Res Commun 495: 325-331, 2018.

43. Wu R, Liu X, Yin J, Wu H, Cai X, Wang N, Qian Y and Wang F: IL-6 receptor blockade ameliorates diabetic nephropathy via inhibiting inflammasome in mice. Metabolism 83: 18-24, 2018.

44. Brouquet A, Benoist S, Julie C, Penna C, Beauchet A, Rougier P and Nordlinger B: Risk factors for chemotherapy-associated liver injuries: A multivariate analysis of a group of 146 patients with colorectal metastases. Surgery 145: 362-371, 2009.

45. Angleton P, Chandler WL and Schmer G: Diurnal variation of tissue-type plasminogen activator and its rapid inhibitor (PAI-1). Circulation 79: 101-106, 1989.

46. Maiello M, Boeri D, Podesta F, Cagliero E, Vichi M, Odetti P, Adezati L and Lorenzi M: Increased expression of tissue plasminogen activator and its inhibitor and reduced fibrinolytic potential of human endothelial cells cultured in elevated glucose. Diabetes 41: 1009-1015, 1992. 
47. Cagliero E, Roth T, Roy S, Maiello M and Lorenzi M: Expression of genes related to the extracellular matrix in human endothelial cells. Differential modulation by elevated glucose concentrations, phorbol esters, and cAMP. J Biol Chem 266: 14244-14250, 1991.

48. Nordt TK, Schneider DJ and Sobel BE: Augmentation of the synthesis of plasminogen activator inhibitor type-1 by precursors of insulin. A potential risk factor for vascular disease. Circulation 89: 321-330, 1994.

49. Alessi MC, Juhan-Vague I, Kooistra T, Declerck PJ and Collen D: Insulin stimulates the synthesis of plasminogen activator inhibitor 1 by the human hepatocellular cell line Hep G2. Thromb Haemost 60: 491-494, 1988.
50. Lijnen HR: Pleiotropic functions of plasminogen activator inhibitor-1. J Thromb Haemostasis 3: 35-45, 2005.

51. Kooistr T: The use of cultured human endothelial cells and hepatocytes as an in vitro model system to study modulation of endogenous fibrinolysis. Fibrinolysis 4 (Suppl 2): S33-S39, 1990.

(i) (9) This work is licensed under a Creative Commons Attribution-NonCommercial-NoDerivatives 4.0 International (CC BY-NC-ND 4.0) License. 